\section{Determinantes do aleitamento materno exclusivo em lactentes menores de seis meses nascidos com baixo peso}

\section{Determinants of exclusive breastfeeding in low birthweight infants under six months of age}

\section{Determinantes de la lactancia materna exclusiva en lactantes menores de seis meses nacidos con bajo peso}

Naiá Ortelan 1

Sonia Isoyama Venancio 2

Maria Helena D’Aquino Benicio 1

doi: $10.1590 / 0102-311 \times 00124618$

\section{Resumo}

Objetivou-se avaliar os fatores associados ao aleitamento materno exclusivo (AME) em lactentes com baixo peso ao nascer, menores de seis meses e residentes em 64 municípios brasileiros. Este estudo transversal incluiu os 2.745 lactentes com baixo peso ao nascer menores de seis meses da Pesquisa de Prevalência de Aleitamento Materno (2008) em municípios brasileiros. $O$ desfecho foi "AME". Os determinantes individuais corresponderam às características socioeconômicas dos lactentes (idade; sexo), das mães (faixa etária; situação de trabalho; paridade) e dos serviços de saúde (nascimento em Hospital Amigo da Criança; local de acompanhamento ambulatorial; tipo de parto). Dois determinantes contextuais foram incluidos: "número de bancos de leite humano por município por 10 mil nascidos vivos", e "IDH do município". O efeito individualizado do fator de estudo sobre o desfecho foi avaliado mediante regressão de Poisson com estrutura multinível, considerando-se associação significante quando $p<5 \%$. O AME foi mais prevalente entre lactentes com baixo peso ao nascer cujas mães tinham de 20-35 anos, não trabalhavam fora ou estavam em licença maternidade; nos que nasceram em Hospital Amigo da Criança e que residiam em municípios com maior número de bancos de leite humano por 10 mil nascidos vivos. O resultado do efeito positivo e independente de estratégias que já integram a Politica Nacional de Aleitamento Materno (nascer em Hospital Amigo da Criança, residir em município com maior disponibilidade de bancos de leite humano e adotar leis que garantam a licença maternidade) sobre o AME em lactentes com baixo peso ao nascer, aponta para a necessidade de expansão da cobertura dessas ações com vistas ao cumprimento das metas de aleitamento propostas pela Organização Mundial da Saúde.

Recém-Nascido de Baixo Peso; Aleitamento Materno; Bancos de Leite Humano

\section{Correspondência}

N. Ortelan

Rua Jerônima Dias 138, apto. 163-B, São Paulo, SP 02407-000, Brasil.

nana.ortelan@gmail.com

1 Faculdade de Saúde Pública, Universidade de São Paulo, São Paulo, Brasil.

2 Instituto de Saúde, Secretaria de Estado da Saúde de São Paulo, São Paulo, Brasil. 


\section{Introdução}

Ao se referir à saúde da criança, o baixo peso ao nascer - definido como a condição de nascido vivo com peso inferior a 2.500 gramas (g) -, e as práticas de amamentação, especificamente o aleitamento materno exclusivo (AME), são questões que modulam, de forma isolada ou em conjunto, os principais desfechos referentes à sobrevivência e desenvolvimento infantis, estendendo-se para o desempenho do processo saúde-doença em médio e longo prazos 1. Por essa lógica, as principais ações de promoção, proteção e apoio ao aleitamento materno que afetam, direta ou indiretamente, crianças nascidas com baixo peso foram tratadas neste estudo.

As principais causas do baixo peso ao nascer são o nascimento pré-termo, o retardo do crescimento intrauterino e a desnutrição fetal 2. A determinação desses processos envolve um conjunto comum de fatores, entre os quais se destacam condições socioeconômicas precárias, baixo peso da mãe no início da gestação, doenças, tabagismo e estresse durante a gestação, falta ou deficiência da assistência pré-natal, antecedentes reprodutivos desfavoráveis e a ocorrência de gravidez múltipla 3.

Algumas estratégias têm contribuído significativamente para aumentar a prevalência do aleitamento materno e AME no Brasil. A Iniciativa Hospital Amigo da Criança (IHAC), implementada pelo Ministério da Saúde em 1992, tem como objetivo garantir apoio das maternidades ao aleitamento materno por meio dos "Dez Passos para o Sucesso do Aleitamento Materno", envolvendo ações relacionadas à organização do processo de trabalho na atenção ao parto e ao puerpério, à capacitação dos profissionais para apoio ao aleitamento durante a estadia no hospital e à promoção do aleitamento materno junto às mães ${ }^{4}$. Apesar de carecer de ampla cobertura no país, tem tido papel importante no aumento da duração do aleitamento materno, no início precoce do aleitamento e na proteção ao AME logo após o nascimento e ao longo dos primeiros meses de vida do lactente 5,6. O desenvolvimento de uma ampla Rede Brasileira de Bancos de Leite Humano, estabelecida em 1998 por iniciativa do Ministério da Saúde e da Fundação Oswaldo Cruz, tem como missão coletar e distribuir leite humano com qualidade certificada e contribuir para a diminuição da mortalidade infantil, além de oferecer atendimento de orientação, palestras e treinamento 7,8. A inclusão, na Constituição Federal Brasileira, do direito a 120 dias de licença-maternidade e sua prorrogação para 180 dias no caso de servidoras públicas 9,10, e a instalação de salas de apoio à amamentação nas empresas 11 são efetivas em aumentar a prática da amamentação exclusiva nos primeiros seis meses de vida da criança.

Recente estudo de tendência temporal com dados secundários de inquéritos nacionais de base populacional (realizados em 1986, 1996, 2006 e 2013), elaborado por Boccolini et al. 12, verificou que a prevalência do AME entre os menores de seis meses aumentou 34,2 pontos percentuais entre 1986 e 2006, indo de 2,9\% para 37,1\%, com ganhos estatisticamente significativos em cada década até 2006 e estabilização em 2013. O acréscimo nas prevalências de AME diminuiu com o aumento da faixa-etária. Entre 1986 e 2006, houve aumento de 44\% na prevalência de AME entre os bebês de zero a dois meses de idade e de 28,1\% entre bebês de três a cinco meses. Em 2013, houve redução da prevalência de AME de 0,3\% entre os bebês de zero a dois meses de idade e 15,1 pontos percentuais entre os bebês de três a cinco meses de idade em relação ao inquérito de 2006.

A II Pesquisa de Prevalência de Aleitamento Materno (PPAM) 13 possibilitou conhecer a situação da amamentação e analisar sua evolução na última década nas capitais brasileiras e Distrito Federal. O AME sofreu considerável expansão desde a década de 1980 e a comparação entre os inquéritos sugere que a velocidade de aumento vem se mantendo estável 9 . Entretanto, não há dados nacionais sobre os determinantes da amamentação exclusiva em nascidos com baixo peso, que constituem o grupo populacional mais vulnerável à mortalidade infantil. Dessa forma, este estudo tem como objetivo verificar os fatores associados ao AME em lactentes nascidos com baixo peso, menores de seis meses residentes em 64 municípios brasileiros. 


\section{Métodos}

Este estudo transversal é parte da PPAM (2008) em municípios brasileiros, que incluiu as 26 capitais, Distrito Federal e 37 municípios com população de crianças menores de um ano superior a 4 mil.

A PPAM objetivou avaliar a situação da amamentação e da alimentação complementar no Brasil. Para atingir maiores coberturas populacionais, os dados foram coletados durante a Campanha Nacional de Vacinação de 2008, utilizando um questionário com questões fechadas sobre características sociodemográficas das mães/responsáveis e dos lactentes, além de informação sobre a prática da amamentação exclusiva para os menores de seis meses de vida. A utilização de dados atuais (current status), recomendada pela Organização Mundial da Saúde (OMS) 14, teve por meta minimizar possíveis vieses decorrentes da memória do informante.

Os procedimentos metodológicos da PPAM tiveram por base a experiência do Projeto Amamentação e Municípios (AMAMUNIC) 15,16 e estão descritos a seguir. Considerando que as crianças não estão distribuídas uniformemente nos postos de vacinação (conglomerados), adotou-se o sorteio em dois estágios, com probabilidade proporcional ao tamanho dos conglomerados. No primeiro estágio, foram sorteados os postos de vacinação e, no segundo, as crianças em cada posto, de forma sistemática. Os postos maiores tiveram maior probabilidade de ser sorteados no primeiro estágio, e as crianças dos postos menores tiveram maior probabilidade de sorteio no segundo estágio. Utilizou-se o estimador deff (efeito de delineamento) como estratégia de validação da técnica amostral, verificando-se se as variâncias obtidas pela amostra por conglomerados, sorteada em dois estágios, seriam diferentes se a amostra fosse casual simples ${ }^{17}$. As amostras para cada município foram planejadas com base nas informações fornecidas pelos estados da federação: número de postos de vacinação em cada município e número de crianças menores de 1 ano vacinadas em cada posto em 2007. Para o cálculo do tamanho amostral, levou-se em conta a prevalência de AME em menores de seis meses das capitais brasileiras e Distrito Federal obtida na I PPAM em 1999 (26,7\%), porém, como se presumia uma melhora deste indicador, ou seja, um aumento da prevalência de AME entre 1999 e 2008, considerou-se um acréscimo de 2 a 10\% no valor da prevalência. Para compensar as perdas de precisão inerentes à amostra por conglomerados, acrescentou-se o efeito do desenho, multiplicando-se por 1,5 a estimativa inicial e, ainda, uma taxa de não resposta de 5 a 10\%. O tamanho da amostra desejado para estimar o AME em menores de 6 meses foi multiplicado por 2, dado que a população-alvo da pesquisa abrangia todas as crianças menores de um ano. Maiores detalhes sobre o cálculo do tamanho amostral e sobre a coleta de dados podem ser obtidos em outras publicações 11,13,15,16.

A Figura 1 apresenta o fluxograma com as definições da população deste estudo. Decidiu-se trabalhar apenas com a população de crianças nascidas com baixo peso da PPAM 2008, em municípios brasileiros, cuja prevalência é equivalente a 8,3\%, para explorar o perfil e os diferenciais dessa população vulnerável com amostra representativa nacional, visando diferenciar este estudo dos demais, pois definiu-se uma população de estudo e não uma variável. Assim, a originalidade está centrada na ênfase ao baixo peso ao nascer e não em mais um estudo de prevalência "baixo peso ao nascer versus não baixo peso ao nascer". Foram excluídos os missings $(\mathrm{n}=3.371)$ para a variável peso ao nascer e também os valores de peso ao nascer considerados inconsistentes $(<500 \mathrm{~g}, \mathrm{n}=72)$. Ao abranger o tema "AME", foi conveniente excluir os lactentes com idade igual ou superior a 6 meses (devido à recomendação de AME até os seis meses). Dessa forma, a população total do estudo foi composta por 2.745 lactentes nascidos com baixo peso, menores de seis meses e residentes em 64 municípios brasileiros.

Foram utilizados procedimentos específicos para análise de inquéritos com amostragem probabilística complexa 11,13,14,18. As estimativas de prevalência e seus respectivos intervalos de $95 \%$ de confiança (IC95\%) foram calculados tendo em consideração o delineamento complexo da amostra e seus respectivos pesos amostrais. Devido às diferenças populacionais entre os municípios estudados, a amostra resultante em cada município correspondeu a uma fração amostral diferente, representada pelo tamanho estimado da amostra sobre o número de crianças a serem vacinadas. $\mathrm{O}$ inverso dessa fração foi aplicado como peso das crianças em cada município para a ponderação do cálculo das prevalências de aleitamento 11,13 .

É importante ressaltar que foi realizada análise de sensibilidade para o desfecho AME, incluindo somente lactentes nascidos com peso entre $2.000 \mathrm{~g}$ e $2.499 \mathrm{~g}$ menores de seis meses, tendo resultados semelhantes à população total com peso inferior a $2.500 \mathrm{~g}$. Ou seja, o fato de o AME (< 6 meses) ter 
Figura 1

Fluxograma que representa as definições da população deste estudo.

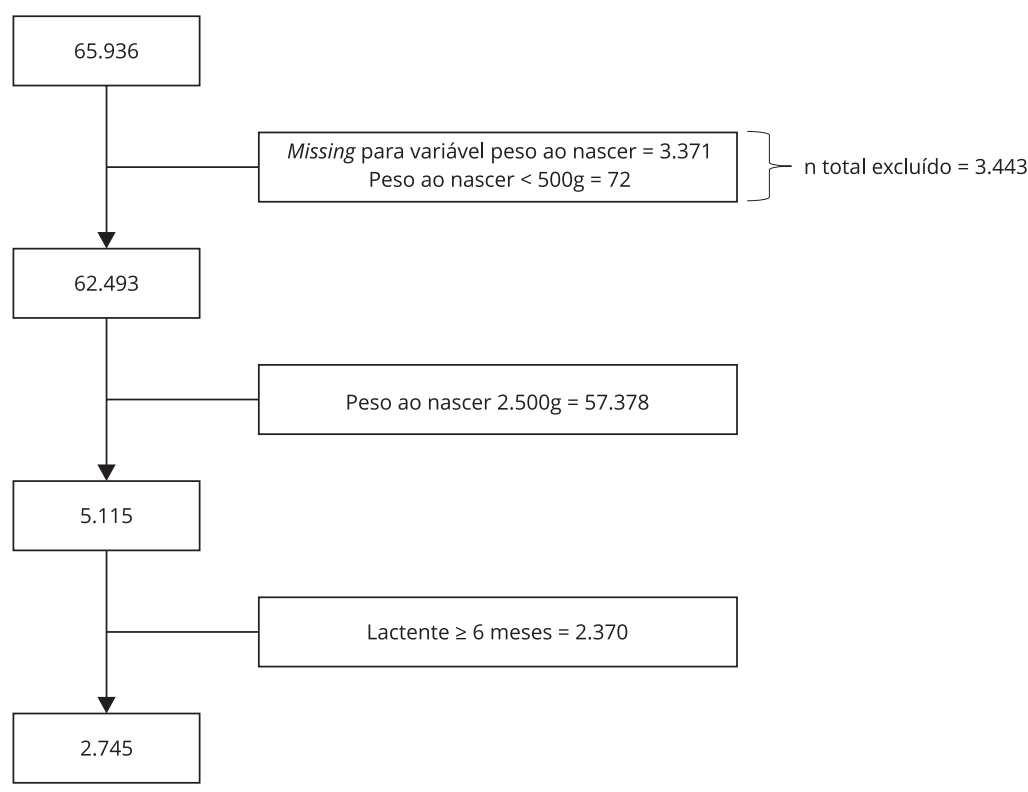

magnitude semelhante nos três estratos de peso ao nascer permitiu que no presente estudo fossem incluídos todos os lactentes com baixo peso ao nascer da amostra.

O desfecho do presente estudo "AME", foi classificado seguindo recomendação da OMS 14, correspondendo aos lactentes de 0-6 meses que receberam somente leite materno no dia anterior à pesquisa, sem nenhum outro líquido ou sólido, com exceção de soro de reidratação oral, medicamentos, xaropes e suplementos vitamínicos ou minerais, sendo construído dicotomicamente: $0=$ não/1 = sim. As variáveis explanatórias corresponderam às características socioeconômicas (representadas pela proxy escolaridade materna, em anos: $\leq 8,9 \mid-12$, $\geq 12$ ), dos lactentes (idade, em dias: $\leq 30,31-60,61-90$, 91-120, 121-150, 151-180; sexo: masculino, feminino), das mães (faixa etária, em anos: < 20, 20|-35, $\geq 35$; situação de trabalho: trabalha fora, não trabalha fora, está em licença maternidade; parição: primípara, multípara) e dos serviços de saúde (nascimento em Hospital Amigo da Criança: não, sim; local de acompanhamento ambulatorial: serviço particular/convênio, rede pública; tipo de parto: normal, cesárea), que constituem os determinantes individuais (Nível 1). Além disso, foram incluídos dois determinantes contextuais (Nível 2): (i) o "número de bancos de leite humano (BLH) por município por 10 mil nascidos vivos”, variável que expressa a razão entre o número de bancos de leite existentes em cada município estudado (informação obtida pela página eletrônica da Rede Brasileira de Bancos de Leite Humano para o ano de 2007) e o número de nascidos vivos no município em 2007 (informação obtida pela página eletrônica do Sistema de Informações sobre Nascidos Vivos - SINASC: http:// tabnet.datasus.gov.br/cgi/deftohtm.exe?sinasc/cnv/nvbr.def), multiplicado por $10 \mathrm{mil}$ (por conta de a razão obtida possuir muitas casas decimais). A ausência de BLH foi representada pela categoria zero (0) - referência. Com isso, o intervalo da razão obtida variou de 0,24 a 4,53, cuja diferença $(4,29)$ foi dividida por três para estimar o valor a ser somado para dar sequência às categorias. Ou seja: 4,29/3= 1,43. Assim, a categoria 1 dessa variável abrangeu os valores entre 0,24 e 1,67 (0,24+1,43); a categoria 2 abrangeu os valores entre 1,68 e 3,10 (1,67+1,43); e a categoria 3, acima de 3,10 (até o valor máximo = 4,53). Portanto, a variável ficou categorizada como segue: $0=0,1=0,24-1,67,2=1,68-3,10,3=$ $\geq 3,11$; e (ii) o Índice de Desenvolvimento Humano (IDH) do município, indicador utilizado para 
referir a condição socioeconômica dos 64 municípios estudados em 2010 (informação obtida pela página eletrônica Atlas do Desenvolvimento Humano no Brasil: http://www.atlasbrasil.org.br/2013/ $\mathrm{pt} /$ consulta/) e estratificado em ordem crescente de classificação 19: baixo (0,5-0,599) + médio (0,6$0,699)$, alto $(0,7-0,799)$, muito alto (>0,8).

O modelo conceitual utilizado está representado na Figura 2. Nele, observa-se que a estratégia de modelagem hierarquizada foi utilizada para inclusão das variáveis individuais no modelo. Nessa estratégia, a hierarquização das variáveis independentes é estabelecida num marco conceitual, e a escolha de critérios para selecioná-las requer conhecimentos sobre precedência temporal, determinantes biológicos e sociais, não considerando somente o aspecto estatístico. A hierarquização foi mantida durante toda a análise, possibilitando a seleção das variáveis mais fortemente associadas ao desfecho de interesse 20 .

A associação entre as variáveis explanatórias e o desfecho foi avaliada inicialmente utilizando-se regressão de Poisson com ajuste apenas pela idade do lactente. $\mathrm{O}$ estudo de associação entre o desfecho e os fatores da análise utilizou modelos multinível de regressão de Poisson. A análise multinível foi usada por se tratar de um estudo que considera dois tipos de variáveis explanatórias (individuais e contextuais) para analisar os determinantes do AME em nascidos com baixo peso e também em função da organização hierárquica das variáveis: àquelas relacionadas às características socioeconômicas, dos lactentes, maternas e aos serviços de saúde (Nível 1) e àquelas relacionadas aos municípios em que as crianças residem (Nível 2), além da existência de correlação intragrupo 21 . Optou-se pela regressão de Poisson por produzir boas estimativas pontuais e intervalares de razão de prevalência (RP) e por ser uma das melhores alternativas para estudos transversais com desfechos binários 22 .

\section{Figura 2}

Marco conceitual para investigação dos determinantes individuais e contextuais do aleitamento materno exclusivo.

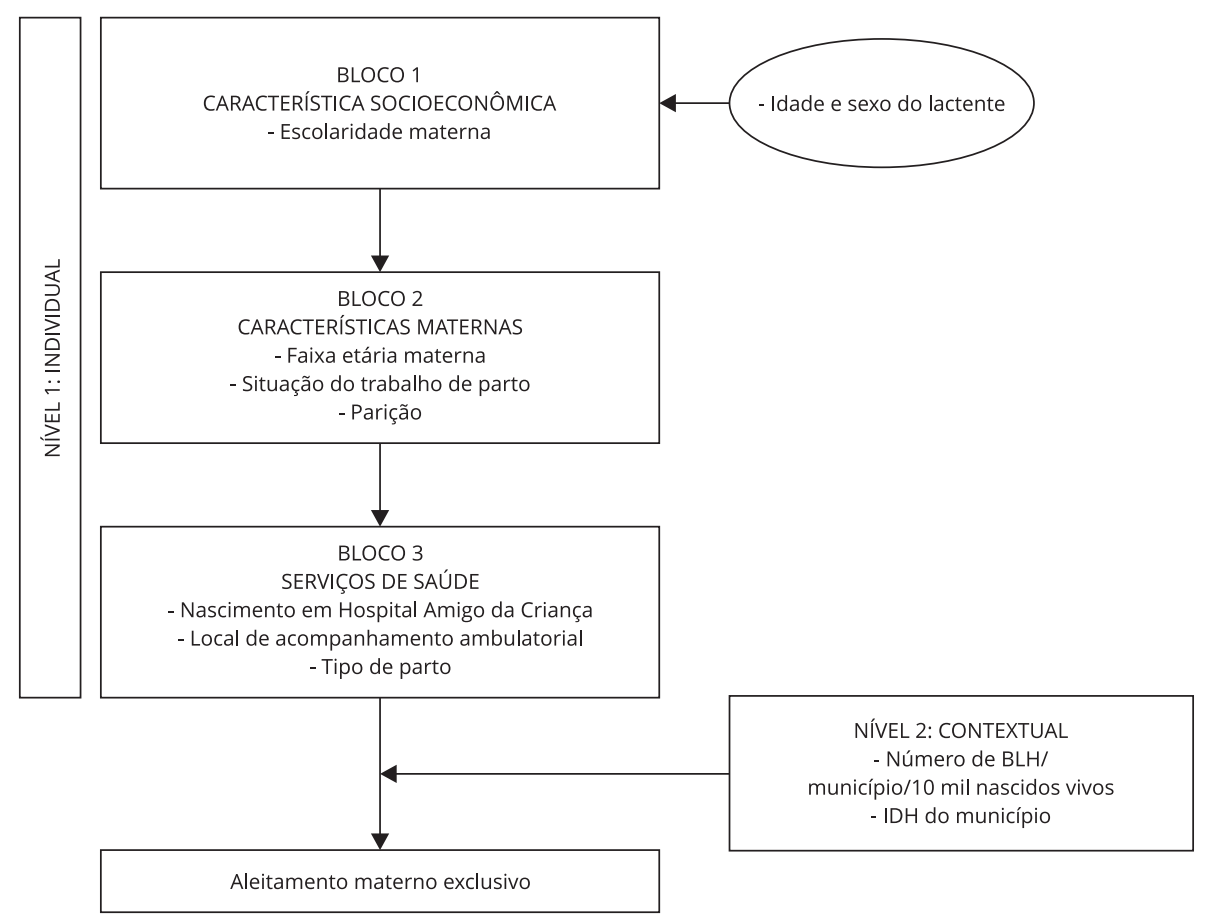

BLH: Banco de Leite Humano; IDH: Índice de Desenvolvimento Humano. 
No modelo final, permaneceram como variáveis de ajuste apenas aquelas que apresentaram $\mathrm{p}<$ 0,15 na análise ajustada pela idade do lactente. A seleção das variáveis que compuseram o modelo final seguiu o processo de seleção backward: no primeiro estágio da modelagem foi inserida a variável escolaridade materna ajustada pela idade do lactente (Bloco 1); posteriormente foram inseridas, respectivamente, àquelas relacionadas às características maternas (Bloco 2) e aos serviços de saúde (Bloco 3) - tais blocos (1, 2 e 3) pertencem ao Nível 1 - e, por último, foram incluídas no modelo as variáveis referentes ao contexto - Nível 2. Variáveis com mais de duas categorias foram introduzidas no modelo em formato dummy para estimar o valor de p de tendência linear. Foram apresentados valores de RP e seus respectivos IC95\%. Variáveis que apresentaram valores de RP entre 0 e 1 foram interpretadas como fatores que diminuem a prevalência do desfecho, e as RP superiores a 1, como fatores que aumentam sua prevalência. A associação entre o fator de estudo e o desfecho foi considerada estatisticamente significante quando $\mathrm{p}<5 \%$. A avaliação da qualidade de ajuste do modelo multinível foi verificada pelo cálculo da desviância (deviance ou -2 log likelihood). Adicionalmente, efetuou-se o teste da razão de verossimilhança para comparar a significância dos modelos de regressão múltipla. Utilizou-se modelo multinível somente com intercepto randômico, conforme descrito por Snijders \& Bosker 23: assume-se que a medida do efeito dos fatores individuais (b1, b2...) é constante em todos os contextos (porção fixa), e que toda variação de ordem contextual recai sobre o intercepto (b0). O processamento foi realizado com o software Stata/SE 14.1 (https://www.stata.com).

Este projeto foi aprovado pelo Comitê de Ética em Pesquisa da Faculdade de Saúde Pública da Universidade de São Paulo, sob parecer número 58147216.1.0000.5421, e seguiu os preceitos éticos estabelecidos conforme a Resolução no 466, de 12 de dezembro de 2012 do Conselho Nacional de Saúde, que regulamenta pesquisas envolvendo seres humanos.

\section{Resultados}

No momento da entrevista, 43,9\% $(\mathrm{n}=911)$ dos lactentes de 0-6 meses com baixo peso ao nascer estavam em AME. A Tabela 1 mostra a caracterização da população estudada. Quanto à característica socioeconômica, 47,6\% das mães tinham entre 9 e 12 anos de escolaridade. Em relação aos lactentes, $55,6 \%$ eram do sexo feminino; a mediana de idade foi três meses e do peso ao nascer, 2.240 gramas (g). No que se refere às características maternas, 65,3\% das mães tinham entre 20 e 35 anos, 66,4\% não trabalhavam fora e 52,5\% eram primíparas. Em relação aos serviços de saúde, 61,7\% dos lactentes não nasceram em Hospital Amigo da Criança; a rede pública foi o principal local de acompanhamento ambulatorial (73\%); e 52,8\% das crianças nasceram de parto cesáreo. No que diz respeito aos determinantes contextuais, a maior parte dos lactentes (42,2\%) residia nos 27 municípios com "número de BLH por 10 mil nascidos vivo" entre 0,24 e 1,67 e mais da metade das crianças $(62,5 \%)$ residia em municípios com alto índice de desenvolvimento humano.

Os resultados da análise ajustada somente pela idade dos lactentes estão descritos na Tabela 2. Lactentes com baixo peso ao nascer cujas mães tinham escolaridade igual ou superior a 12 anos, idade entre 20 e 35 anos e que estavam em licença maternidade apresentaram maior prevalência de aleitamento materno exclusivo. O mesmo se deu para os nascidos em Hospital Amigo da Criança e em municípios com maior número de BLH por 10 mil nascidos vivos.

A Tabela 3 apresenta os resultados da análise multinível. O modelo final de regressão múltipla apresentou qualidade de ajuste significante mais elevada, conforme indicado pelo teste da razão de verossimilhança. Após ajuste para as demais variáveis, o aleitamento materno exclusivo foi mais prevalente entre lactentes nascidos com baixo peso cujas mães tinham de 20-35 anos de idade $(\mathrm{RP}=$ 1,35; IC95\%: 1,09-1,69) e não trabalhavam fora ( $R P=1,36$; IC95\%: 1,08-1,71) ou que estavam em licença maternidade ( $\mathrm{RP}=1,30$; IC95\%: 1,06-1,59); lactentes que nasceram em Hospital Amigo da Criança ( $R P=1,22$; IC95\%: 1,09-1,37) e que residiam em municípios com maior número de BLH

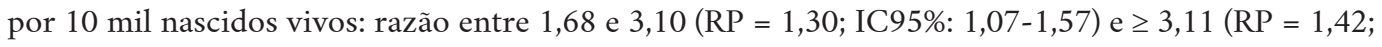
IC95\%: 1,14-1,76). 


\section{Tabela 1}

Características dos lactentes nascidos com baixo peso menores de seis meses e dos municípios estudados. Pesquisa de Prevalência de Aleitamento Materno em municípios brasileiros, 2008 ( $\mathrm{N}=2.745)$.

\begin{tabular}{|c|c|c|}
\hline Variável & Número de lactentes & $\%$ \\
\hline \multicolumn{3}{|c|}{ Bloco 1: característica socioeconômica } \\
\hline \multicolumn{3}{|l|}{ Escolaridade materna (anos) } \\
\hline$\leq 8$ & 1.122 & 42,2 \\
\hline $9-12$ & 1.083 & 47,6 \\
\hline$\geq 12$ & 280 & 10,2 \\
\hline \multicolumn{3}{|c|}{ Bloco 2: características dos lactentes e maternas } \\
\hline \multicolumn{3}{|l|}{ Sexo } \\
\hline Masculino & 1.231 & 44,4 \\
\hline Feminino & 1.514 & 55,6 \\
\hline Idade dos lactentes (meses) & 3 * & $1,6-4,5 * *$ \\
\hline Peso ao nascer (g) & 2.240 * & $2.005-2.380$ ** \\
\hline$<1.500$ & 188 & 6,0 \\
\hline $1.500-1.999$ & 445 & 15,3 \\
\hline $2.000-2.499$ & 2.112 & 78,7 \\
\hline \multicolumn{3}{|l|}{ Faixa etária materna (anos) } \\
\hline$<20$ & 567 & 21,3 \\
\hline $20-35$ & 1.668 & 65,3 \\
\hline$\geq 35$ & 269 & 13,4 \\
\hline \multicolumn{3}{|l|}{ Trabalho materno } \\
\hline Trabalha fora & 341 & 14,7 \\
\hline Não trabalha fora & 1.660 & 66,4 \\
\hline Está em licença maternidade & 417 & 18,9 \\
\hline \multicolumn{3}{|l|}{ Parição } \\
\hline Primípara & 1.289 & 52,5 \\
\hline Multípara & 1.176 & 47,5 \\
\hline \multicolumn{3}{|l|}{ Bloco 3: serviços de saúde } \\
\hline \multicolumn{3}{|c|}{ Nascimento em Hospital Amigo da Criança } \\
\hline Não & 1.681 & 61,7 \\
\hline Sim & 879 & 38,3 \\
\hline \multicolumn{3}{|c|}{ Local de acompanhamento ambulatorial } \\
\hline Serviço particular/Convênio & 569 & 27,0 \\
\hline Rede pública & 1.572 & 73,0 \\
\hline \multicolumn{3}{|l|}{ Tipo de parto } \\
\hline Normal & 1.262 & 47,2 \\
\hline \multirow[t]{2}{*}{ Cesárea } & 1.464 & 52,8 \\
\hline & Número de municípios & $\%$ \\
\hline
\end{tabular}

\section{Bloco 4: determinantes contextuais}

Número de BLH/10.000 nascidos vivos

\begin{tabular}{|c|c|c|}
\hline 0 & 28 & 43,7 \\
\hline $0,24-1,67$ & 27 & 42,2 \\
\hline $1,68-3,10$ & 8 & 12,5 \\
\hline$\geq 3,11$ & 1 & 1,6 \\
\hline \multicolumn{3}{|l|}{ DH do município } \\
\hline Baixo $(0,5-0,599)+$ médio $(0,6-0,699)$ & 14 & 21,9 \\
\hline Alto $(0,7-0,799)$ & 40 & 62,5 \\
\hline Muito alto $(>0,8)$ & 10 & 15,6 \\
\hline
\end{tabular}

BLH: Banco de Leite Humano; IDH: Índice de Desenvolvimento Humano.

* Mediana;

** Intervalo interquartil. 
Tabela 2

Fatores associados ao aleitamento materno exclusivo e respectivas razões de prevalência (RP) ajustadas pela idade dos lactentes menores de seis meses com baixo peso ao nascer. Pesquisa de Prevalência de Aleitamento Materno em municípios brasileiros, 2008.

\begin{tabular}{|c|c|c|c|c|c|}
\hline \multirow[t]{2}{*}{ Variável } & \multicolumn{5}{|c|}{ Aleitamento materno exclusivo } \\
\hline & n & $\operatorname{Sim}(\%)$ & Valor de $p$ & RP * (IC95\%) & Valor de $p$ \\
\hline \multicolumn{6}{|l|}{ Bloco 1: característica socioeconômica } \\
\hline \multicolumn{6}{|l|}{ Escolaridade materna (anos) } \\
\hline$\leq 8$ & 900 & 42,8 & & 1,00 & \\
\hline $9-12$ & 823 & 45,1 & 0,587 & $1,08(0,89-1,32)$ & $0,134 * *$ \\
\hline$\geq 12$ & 195 & 48,8 & & $1,16(0,97-1,38)$ & \\
\hline \multicolumn{6}{|c|}{ Bloco 2: características dos lactentes e maternas } \\
\hline \multicolumn{6}{|l|}{ Sexo } \\
\hline Masculino & 926 & 45,2 & 0,566 & 1,00 & \\
\hline Feminino & 1.161 & 42,9 & & $0,95(0,79-1,14)$ & 0,566 \\
\hline \multicolumn{6}{|l|}{ Faixa etária materna (anos) } \\
\hline$<20$ & 451 & 33,7 & & 1,00 & \\
\hline $20-35$ & 1.290 & 49,1 & 0,024 & $1,35(1,10-1,67)$ & $0,060 * *$ \\
\hline$\geq 35$ & 189 & 41,6 & & $1,29(0,91-1,74)$ & \\
\hline \multicolumn{6}{|l|}{ Trabalho materno } \\
\hline Trabalha fora & 236 & 29,1 & $<0,001$ & 1,00 & \\
\hline Não trabalha fora & 1.314 & 46,0 & & $1,29(1,03-1,63)$ & 0,009 ** \\
\hline Está em licença maternidade & 325 & 54,0 & & $1,35(1,08-1,68)$ & \\
\hline \multicolumn{6}{|l|}{ Parição } \\
\hline Primípara & 967 & 42,3 & 0,005 & 1,00 & \\
\hline Multípara & 934 & 48,1 & & $1,08(0,97-1,21)$ & 0,165 \\
\hline \multicolumn{6}{|l|}{ Bloco 3: serviços de saúde } \\
\hline \multicolumn{6}{|l|}{ Nascimento em Hospital Amigo da Criança } \\
\hline Não & 1.268 & 39,0 & $<0,001$ & 1,00 & \\
\hline Sim & 682 & 51,0 & & $1,23(1,15-1,32)$ & $<0,001$ \\
\hline \multicolumn{6}{|l|}{ Local de acompanhamento ambulatorial } \\
\hline Serviço particular/Convênio & 386 & 39,6 & 0,095 & 1,00 & \\
\hline Rede pública & 1.234 & 45,0 & & $1,05(0,90-1,22)$ & 0,513 \\
\hline \multicolumn{6}{|l|}{ Tipo de parto } \\
\hline Vaginal & 1.014 & 41,2 & 0,337 & 1,00 & \\
\hline Cesárea & 1.060 & 46,7 & & $1,14(0,91-1,43)$ & 0,254 \\
\hline \multicolumn{6}{|l|}{ Bloco 4: determinantes contextuais } \\
\hline \multicolumn{6}{|l|}{ Número de BLH/10.000 nascidos vivos } \\
\hline 0 & 748 & 41,7 & 0,026 & 1,00 & \\
\hline $0,24-1,67$ & 1.027 & 42,5 & & $1,04(0,86-1,26)$ & \\
\hline $1,68-3,10$ & 266 & 54,2 & & $1,35(1,13-1,62)$ & 0,025 ** \\
\hline$\geq 3,11$ & 46 & 58,7 & & $1,35(1,13-1,62)$ & \\
\hline \multicolumn{6}{|l|}{ IDH do município } \\
\hline Baixo $(0,5-0,599)+$ médio $(0,6-0,699)$ & 309 & 31,7 & 0,214 & 1,00 & \\
\hline Alto $(0,7-0,799)$ & 1.355 & 43,3 & & $1,33(0,94-1,88)$ & 0,120 \\
\hline Muito alto $(>0,8)$ & 423 & 45,8 & & $1,45(1,01-2,07)$ & \\
\hline
\end{tabular}

BLH: Banco de Leite Humano; IC95\%: intervalo de 95\% de confiança; IDH: Índice de Desenvolvimento Humano.

Nota: valor de p de tendência linear da idade dos lactentes (em meses): <0,001; valores de $p<0,15$ apresentadas em negrito.

* Valores de RP ajustadas pela idade das crianças;

** Valor de $p$ de tendência linear. 
Tabela 3

Determinantes do aleitamento materno exclusivo em crianças com idade inferior a seis meses com baixo peso ao nascer. Pesquisa de Prevalência de Aleitamento Materno em municípios brasileiros, 2008.

\begin{tabular}{|c|c|c|c|c|}
\hline Variável & $\begin{array}{c}\text { Modelo 0 } \\
(\mathrm{n}=\mathbf{2 . 0 8 7 )} \\
\mathrm{RP}_{\text {ajustada }}(\mathrm{IC95 \% )}\end{array}$ & $\begin{array}{c}\text { Modelo } 1 \\
(\mathrm{n}=1.848) \\
\mathrm{RP}_{\text {ajustada }}(\mathrm{IC} 95 \%)\end{array}$ & $\begin{array}{c}\text { Modelo } 2 \\
(\mathrm{n}=1.754) \\
\mathrm{RP}_{\text {ajustada }}(\mathrm{IC} 95 \%)\end{array}$ & $\begin{array}{c}\text { Modelo } 3 \\
(\mathrm{n}=1.754) \\
\mathrm{RP}_{\text {ajustada }}(\mathrm{IC95 \% )}\end{array}$ \\
\hline Efeitos fixos - constante & $0,65(0,59-0,72)$ & $0,38(0,26-0,54)$ & $0,33(0,24-0,46)$ & $0,27(0,16-0,43)$ \\
\hline \multicolumn{5}{|l|}{ Bloco 1: característica socioeconômica } \\
\hline \multicolumn{5}{|l|}{ Escolaridade materna (anos) } \\
\hline$\leq 8$ & & 1,00 & & \\
\hline $9-12$ & & $1,04(0,89-1,21)$ & & \\
\hline$\geq 12$ & & $1,11(0,93-1,32)$ & & \\
\hline Valor de $\mathrm{p}$ & & 0,538 * & & \\
\hline \multicolumn{5}{|c|}{ Bloco 2: características dos lactentes e maternas } \\
\hline \multicolumn{5}{|l|}{ Faixa etária materna (anos) } \\
\hline$<20$ & & 1,00 & & \\
\hline $20-35$ & & $1,35(1,09-1,69)$ & & \\
\hline$\geq 35$ & & $1,23(0,88-1,70)$ & & \\
\hline Valor de p & & $0,113 *$ & & \\
\hline \multicolumn{5}{|l|}{ Trabalho materno } \\
\hline Trabalha fora & & 1,00 & & \\
\hline Não trabalha fora & & $1,36(1,08-1,71)$ & & \\
\hline Está em licença maternidade & & $1,30(1,06-1,59)$ & & \\
\hline Valor de $\mathrm{p}$ & & 0,023 * & & \\
\hline \multicolumn{5}{|l|}{ Bloco 3: serviços de saúde } \\
\hline \multicolumn{5}{|l|}{ Nascimento em Hospital Amigo da Criança } \\
\hline Não & & & 1,00 & \\
\hline Sim & & & $1,22(1,09-1,37)$ & \\
\hline Valor de $\mathrm{p}$ & & & 0,001 & \\
\hline \multicolumn{5}{|l|}{ Bloco 4: determinantes contextuais } \\
\hline \multicolumn{5}{|l|}{ Número de BLH/10.000 nascidos vivos } \\
\hline 0 & & & & 1,00 \\
\hline $0,24-1,67$ & & & & $1,06(0,88-1,29)$ \\
\hline $1,68-3,10$ & & & & $1,30(1,07-1,57)$ \\
\hline$\geq 3,11$ & & & & $1,42(1,14-1,76)$ \\
\hline Valor de $\mathrm{p}$ & & & & 0,020 * \\
\hline \multicolumn{5}{|l|}{ IDH do município } \\
\hline Baixo $(0,5-0,599)+$ Médio $(0,6-0,699)$ & & & & 1,00 \\
\hline Alto $(0,7-0,799)$ & & & & $1,22(0,88-1,69)$ \\
\hline Muito alto $(>0,8)$ & & & & $1,17(0,84-1,63)$ \\
\hline Valor de p & & & & 0,822 * \\
\hline Desviância (-2 log likelihood) & 3211,2272 & 2875,6294 & 2729,8104 & 2719,7602 \\
\hline Teste da razão de verossimilhança & & $335,5978(p<0,001)$ & $145,8190(p<0,001)$ & $10,0502(p=0,007)$ \\
\hline
\end{tabular}

BLH: Banco de Leite Humano; IC95\%: intervalo de 95\% de confiança; IDH: Índice de Desenvolvimento Humano; RP: razões de prevalência. Nota: modelo 0: idade dos lactentes; modelo 1: modelo 0 + escolaridade, faixa etária e trabalho maternos; modelo 2: modelo 1 + nascimento em Hospital Amigo da Criança; modelo 3: modelo 2 + nascimento em município com BLH/10 mil nascidos vivos e IDH do município.

* Valor de $p$ de tendência linear. 


\section{Discussão}

Este é o primeiro estudo de abrangência nacional que explora a relação de determinantes individuais e contextuais com a prevalência de amamentação exclusiva em lactentes nascidos com baixo peso. E, apesar de essa população vulnerável apresentar características biológicas e sociais diferentes das crianças em geral, constatou-se que os fatores que influenciam a prática de AME são semelhantes para ambas 13 .

As práticas de amamentação são afetadas por uma ampla gama de fatores históricos, socioeconômicos, culturais e individuais. $\mathrm{O}$ trabalho materno é um dos principais motivos para a interrupção da amamentação ou para o desmame precoce, em razão de seu efeito multidimensional, que inclui fadiga, praticidade (visto que oferecer leite não materno é mais prático do que amamentar após o retorno ao trabalho) e intensidade (número de horas trabalhadas) 24,25 . O presente estudo constatou o efeito desfavorável do trabalho na amamentação exclusiva de lactentes com baixo peso ao nascer, corroborando com Monteiro et al. 26 que, ao analisar dados da II PPAM e de inquérito realizado em Brasília, mostrou que a ausência de licença maternidade aumentou em $23 \%$ a chance de interrupção do AME, evidenciando o efeito protetor da licença maternidade em relação ao AME para todos os lactentes menores de seis meses.

O número crescente de mulheres no mercado de trabalho chama a atenção para a importância dos intervalos durante o expediente, em salas próprias para amamentação no local de trabalho e para a provisão da licença maternidade 27 . No entanto, poucas mulheres são adequadamente apoiadas durante a amamentação por direitos da maternidade e do trabalho para serem capazes de trabalhar ou frequentar a escola e ainda amamentarem; muitas vezes, tais direitos ou não são fornecidos, ou as mulheres que trabalham na economia informal não são elegíveis. Embora quase todos os países tenham legislação que protege a maternidade, apenas 98 (53\%) dos 185 países atingiram o padrão mínimo da Organização Mundial do Trabalho de 14 semanas e somente 42 (23\%) atingiram ou superaram a recomendação de licença de 18 semanas 25 . Evidências sugerem que políticas de licença maternidade são efetivas em aumentar o AME 26,27. Em contrapartida, mulheres que planejam retornar ao trabalho após o parto têm menor probabilidade de iniciar ou continuar a amamentação 28 . A licença maternidade curta (< 6 semanas) leva a um aumento de quatro vezes a chance de não iniciar a amamentação ou de desmame precoce 27 . Estudo realizado em todos os estados membros das Nações Unidas verificou que a garantia de licença maternidade paga por pelo menos 6 meses foi associada com um aumento de 8,9 pontos percentuais na amamentação exclusiva 29. Outro estudo também realizado nos Estados Unidos mostrou que salas de aleitamento e pausas para ordenha do leite materno aumentaram a amamentação aos 6 meses em 25\% 30. A redução das barreiras para mães trabalhadoras amamentarem por meio da provisão de salas de aleitamento e pausas para amamentar são intervenções de baixo custo que podem reduzir o absenteísmo e melhorar o desempenho, o comprometimento e a retenção da força de trabalho 27 .

Apesar de a prevalência de AME de 43,9\% obtida neste estudo ser classificada como "razoável" (entre 12\% e 49\%) segundo parâmetros propostos pela OMS 31, é considerada muito boa quando comparada aos dados das crianças em geral ${ }^{13}$. O fato de os lactentes que nasceram com baixo peso em Hospital Amigo da Criança terem a razão de prevalência de AME aumentada em 22\% em relação aos que não nasceram em Hospital Amigo da Criança, destaca o impacto positivo dessa iniciativa na amamentação exclusiva da população vulnerável. Embora a IHAC seja voltada a bebês sadios e a termo, a implantação dos "Dez Passos para o Sucesso do Aleitamento Materno" parece exercer também influência sobre os nascidos com baixo peso.

No Brasil, foi relatado um significativo aumento das taxas de aleitamento materno e, especialmente, de AME entre as mães atendidas nos Hospitais Amigos da Criança 32. Bicalho-Mancini \& Velásquez-Meléndez 33 demonstraram que a taxa de AME à alta hospitalar mudou de 36 para 54,6\%, após uma maternidade de Minas Gerais aderir à IHAC. Em nível internacional, estudo randomizado incluindo 31 hospitais em Belarus, revelou que a intervenção baseada nos princípios da IHAC aumentou as taxas do aleitamento materno, sobretudo do aleitamento materno exclusivo 12. Na Escócia, bebês nascidos em hospitais com o status de amigo da criança tiveram $28 \%$ mais probabilidade de serem amamentados exclusivamente ao seio do que àqueles que nasceram em outras maternidades 34. Apesar de esta iniciativa ser um importante elemento na política de apoio ao aleitamento materno, 
mais esforços são necessários para aumentar o número de hospitais credenciados no Brasil, o que implica mudanças nas atuais exigências e uma política de maior investimento pelo setor público.

A prevalência da amamentação sofre influência não só das características das crianças, das mães e dos serviços de saúde, mas também da existência de programas e ações pró-amamentação nos municípios 35,36. O presente estudo revela que o AME foi mais prevalente entre lactentes que residiam em municípios com maior número de BLH por 10 mil nascidos vivos, fortalecendo essa evidência.

O modelo brasileiro de BLH é reconhecido mundialmente pelo desenvolvimento tecnológico inédito que alia baixo custo à alta qualidade, além de distribuir o leite humano conforme as necessidades específicas de cada bebê (inclusive prematuros e de baixo peso), realizar atendimento de orientação e apoio à amamentação, aumentando a eficácia da iniciativa para a redução da mortalidade neonatal. Em 2001, a OMS reconheceu a Rede Brasileira de BLH como uma das ações que mais contribuíram para redução da mortalidade infantil no mundo na década de 1990. De 1990 a 2012, a taxa de mortalidade infantil no Brasil reduziu 70,5\% 37. Os BLH estão distribuídos em todos os estados do território nacional, alguns com coleta domiciliar. Tais avanços continuam sendo de extrema importância para o Brasil alcançar o terceiro Objetivo de Desenvolvimento Sustentável até 2030: reduzir as mortes evitáveis de crianças menores de cinco anos para até 25 por mil nascidos vivos 37 .

A literatura tem evidenciado que não basta apenas a abordagem hospitalar para o sucesso do aleitamento materno, sendo necessário apoio dos profissionais de saúde durante o processo de amamentação e estratégias após a alta hospitalar, com formação de grupos de apoio 8,36 . O contato frequente e contínuo com grupos promotores de aleitamento materno (BLH, visitas domiciliares) mostra-se útil no combate ao desmame precoce, com proporção significativamente maior do AME 8,38,39,40.

Nessa perspectiva, no ano 2000 o Ministério da Saúde lançou a Norma de Atenção Humanizada ao Recém-Nascido de Baixo Peso - Método Canguru, como política nacional de saúde, sendo uma alternativa para estimular a alta hospitalar precoce e promover maior duração do aleitamento materno nos recém-nascidos de baixo peso estáveis clinicamente 41. Em 2015, lançou o manual do Método Canguru na atenção básica 42 para apoiar os profissionais de saúde na atenção ao nascido de baixo peso egresso de unidade neonatal, qualificando o cuidado e promovendo uma articulação em rede. Infelizmente, não foram disponibilizadas informações a respeito da implantação do Método Canguru para o ano de 2007 nos hospitais onde os lactentes deste estudo nasceram, o que poderia enriquecer nossos achados.

O conhecimento, baseado em evidências, dos fatores associados à interrupção do AME de lactentes nascidos com baixo peso, tem servido de apoio para que ações, políticas locais e programas promovam o aleitamento materno mediante ações assistenciais prestadas às mulheres, incluindo o apoio psicológico e sociocultural, uma vez que a maioria dos fatores identificados é passível de intervenção 43.

Pesquisas em campanhas de vacinação permitem a obtenção de informações em um curto período e com baixo custo; e a utilização da análise multinível permite a obtenção de estimativas que levam em consideração o nível hierárquico dos dados e a correlação intragrupo 21 . Além disso, a PPAM é o estudo epidemiológico de representatividade para todas as regiões do país mais completo e atual para avaliar a amamentação de lactentes nascidos com baixo peso menores de seis meses, ressaltando os pontos fortes deste estudo. Dentre as limitações, destacam-se: (i) o delineamento transversal do estudo, que não permite estabelecer a procedência temporal entre os fatores de exposição e o desfecho, indispensável para o estabelecimento de relações causais. Contudo, os resultados são úteis para formulação de hipóteses; (ii) o fato de as informações referentes ao AME dos lactentes se referirem somente ao dia anterior ao inquérito, impossibilitando avaliar a frequência da amamentação. No entanto, a utilização de um único recordatório não diminui a validade do estudo, já que se objetivou realizar avaliação de grupo e não da qualidade do aleitamento materno; e (iii) a impossibilidade de analisar a influência do Método Canguru, em função da não disponibilidade de informações sobre a implementação do Método Canguru para o ano analisado.

Estratégias bem-sucedidas de promoção, proteção e apoio à amamentação em lactentes com baixo peso ao nascer necessitam de medidas em diversos níveis, desde legislação, investimento financeiro e políticas, até atitudes e normas sociais, condições de trabalho e emprego para mulheres, e serviços de saúde para apoiar as mulheres e suas famílias na amamentação adequada, contribuindo para reduzir significativamente a mortalidade neonatal, infantil e materna. Por fim, merece destaque o efeito positivo e independente sobre o AME detectado de forma pioneira em crianças com baixo peso ao 
nascer para estratégias que integram a Política Nacional de Aleitamento Materno: nascer em Hospital Amigo da Criança, residir em município com maior disponibilidade de BLH e adotar leis que garantam a licença maternidade. Esse resultado aponta para a necessidade de expansão da cobertura dessas estratégias com vistas ao cumprimento das metas de aleitamento materno propostas pela OMS.

\section{Colaboradores}

N. Ortelan conduziu as análises, a interpretação dos dados, o planejamento e concepção do manuscrito, bem como as correções após considerações dos coautores. S. I. Venancio contribuiu substancialmente com a revisão crítica e correção do conteúdo, aprovando a versão final. M. H. D’A. Benicio foi responsável pela orientação da tese que resultou na elaboração deste manuscrito, pela correção e aprovação da versão final.

\section{Informações adicionais}

ORCID: Naiá Ortelan (0000-0001-6535-748X); Sonia Isoyama Venancio (0000-0001-7147-3292); Maria Helena D’Aquino Benicio (0000-0003-18511178).

\section{Agradecimentos}

Agradeço imensamente ao professor José Leopoldo Ferreira Antunes, membro da minha banca de doutorado que acompanhou toda minha trajetória, pela presteza, prontidão, atenção e ajuda com minhas análises e com as respostas metodológicas aos pareceristas; e à Maria Antonieta de Barros Leite Carvalhaes, pela revisão crítica deste manuscrito durante a arguição da minha tese. Vocês contribuíram com excelência para a concretização deste manuscrito.

\section{Referências}

1. Caminha MFC, Azevedo PTACC, Sampaio BB, Acioly VMC, Belo MPM, Lira PIC, et al. Aleitamento materno em crianças de 0 a 59 meses no Estado de Pernambuco, Brasil, segundo o peso ao nascer. Ciênc Saúde Colet 2014; 19:2021-32.

2. World Health Organization; United Nations Children's Fund. Low birth weight: country, regional and global estimates. New York: United Nations Children's Fund; 2004.

3. Monteiro CA, Benicio MHD’A, Ortiz LP. Tendência secular do peso ao nascer na cidade de São Paulo (1976-1998). Rev Saúde Pública 2000; 34:26-40.

4. Sampaio PF, Moraes CL, Reichenheim ME, Oliveira ASD, Lobato G. Nascer em Hospital Amigo da Criança no Rio de Janeiro, Brasil: um fator de proteção ao aleitamento materno? Cad Saúde Pública 2011; 27:1349-61.

5. World Health Organization; United Nations Children's Fund. Baby-friendly hospital initiative: revised, updated and expanded for integrated care, Section 1, Background and implementation. http://www.unicef.gr/pdfs/ BFHI_2009update.pdf (acessado em 20/Dez/ 2018).

6. Pérez-Escamilla R, Martinez JL, Segura-Pérez S. Impact of the Baby-friendly Hospital Initiative on breastfeeding and child health outcomes: a systematic review. Matern Child Nutr 2016; 12:402-17.

7. Rede Global de Bancos de Leite Humano. https://rblh.fiocruz.br/pagina-inicial-rede-blh (acessado em 20/Dez/2018).

8. Maia PRDS, Almeida JAG, Novak FR, Silva DA. Rede Nacional de Bancos de Leite Humano: gênese e evolução. Rev Bras Saúde Mater Infant 2006; 6:285-92.

9. Venancio SI, Saldiva SRDM, Monteiro CA Tendência secular da amamentação no Brasil. Rev Saúde Pública 2013; 47:1205-8. 
10. Brasil. Decreto no 6690, de 11 de dezembro de 2008. Institui o Programa de Prorrogação da Licença à Gestante e à Adotante, estabelece os critérios de adesão ao Programa e dá outras providências. Diário Oficial da União 2008; 12 dez.

11. Venancio SI, Escuder MML, Saldiva SRDM. Breastfeeding practice in the Brazilian capital cities and the Federal District: current status and advances. J Pediatr (Rio J.) 2010; 86:31724.

12. Boccolini CS, Boccolini PMM, Monteiro FR, Venancio SI, Giugliani ERJ. Tendência de indicadores do aleitamento materno no Brasil em três décadas. Rev Saúde Pública 2017; 51:108.

13. Departamento de Ações Programáticas e Estratégicas, Secretaria de Atenção à Saúde, Ministério da Saúde. II Pesquisa de Prevalência de Aleitamento Materno nas capitais brasileiras e Distrito Federal. Brasília: Ministério da Saúde; 2009. (Série C. Projetos, Programas e Relatórios).

14. Venancio SI, Escuder MML, Kitoko P, Rea MF, Monteiro CA. Frequência e determinantes do aleitamento materno em municípios do Estado de São Paulo. Rev Saúde Pública 2002; 36:313-8.

15. Venancio SI, Saldiva SRDM, Castro ALS, Gouveia AGC, Santana AC, Pinto JCC, et al. Projeto Amamentação e Municípios: a trajetória de implantação de uma estratégia para a avaliação e monitoramento das práticas de alimentação infantil no Estado de São Paulo, no período de 1998-2008. BEPA, Bol Epidemiol Paul 2010; 7:4-15.

16. Silva NN. Amostragem probabilística. São Paulo: EdUSP; 1998.

17. World Health Organization. Indicators for assessing infant and young child feeding practices: conclusions of a consensus meeting held 6-8 November 2007 in Washington D.C., USA. Geneva: World Health Organization; 2009.

18. Saldiva SRDM, Venancio SI, Santana AC, Castro ALS, Escuder MML, Giugliani ERJ. The consumption of unhealthy foods by Brazilian children is influenced by their mother's educational level. Nutr J 2014; 13:33.

19. Programa Nacional das Nações Unidas para o Desenvolvimento. Ranking IDHM Municípios 2010. http://www.br.undp.org/content/ brazil/pt/home/idh0/rankings/idhm-munici pios-2010.html (acessado em 20/Dez/2018).

20. Victora CG, Huttly SR, Fuchs SC, Olinto MT. The role of conceptual frameworks in epidemiological analysis: a hierarchical approach. Int J Epidemiol 1997; 26:224-7.

21. Venancio SI, Monteiro CA. Individual and contextual determinants of exclusive breastfeeding in São Paulo, Brazil: a multilevel analysis. Public Health Nutr 2006; 9:40-6.

22. Barros AJD, Hirakata VN. Alternatives for logistic regression in cross-sectional studies: an empirical comparison of models that directly estimate the prevalence ratio. BMC Med Res Methodol 2003; 3:21.
23. Snijders T, Bosker R. The random intercept model. In: Snijders T, Bosker R, editors. Multilevel analysis: an introduction to basic and applied multilevel analysis. 2nd Ed. London: Sage; 2012. p. 39-61.

24. Ogbuanu C, Glover S, Probst J, Liu J, Hussey J. The effect of maternity leave length and time of return to work on breastfeeding. Pediatrics 2011; 127:e1414-27.

25. Rollins NC, Bhandari N, Hajeebhoy N, Horton S, Lutter CK, Martines JC, et al. Why invest, and what it will take to improve breastfeeding practices? Lancet 2016; 387:491-504.

26. Monteiro FR, Buccini GS, Venancio SI, Costa THM. Influence of maternity leave on exclusive breastfeeding. J Pediatr (Rio J.) 2017; 93:475-81.

27. International Labour Organization. Maternity and paternity at work: law and practice across the world. Geneva: International Labour Organization; 2014.

28. Mirkovic KR, Perrine CG, Scanlon KS, Grummer-Strawn LM. In the United States, a mother's plans for infant feeding are associated with her plans for employment. J Hum Lact 2014; 30:292-7.

29. Heymann J, Raub A, Earle A. Breastfeeding policy: a globally comparative analysis. Bull World Health Organ 2013; 91:398-406.

30. Dabritz H, Hinton B, Babb J. Evaluation of lactation support in the workplace or school environment on 6-month breastfeeding outcomes in Yolo County, California. J Hum Lact 2009; 25:182-93.

31. World Health Organization; United Nations Children's Fund. Global Strategy for Infant and Young Child Feeding. Geneva: World Health Organization; 2003.

32. Lamounier JA, Bouzada MCF, Janneu AMDS, Maranhão AGK, Araújo MDFM, Vieira GO, et al. Iniciativa Hospital Amigo da Criança, mais de uma década no Brasil: repensando o futuro. Rev Paul Pediatr (Ed. Port., Online) 2008; 26:161-9.

33. Bicalho-Mancini PG, Velásquez-Meléndez G. Aleitamento materno exclusivo na alta de recém-nascidos internados em berçário de alto risco e os fatores associados a essa prática. J Pediatr (Rio J.) 2004; 80:241-8.

34. Broadfoot M, Britten J, Tappin D, MacKenzie J. The Baby Friendly Hospital Initiative and breastfeeding rates in Scotland. Arch Dis Child Fetal Neonatal Ed 2005; 90:F114-6.

35. Venancio SI, Saldiva SRDM, Escuder MML, Giugliani ERJ. The Baby-Friendly Hospital Initiative shows positive effects on breastfeeding indicators in Brazil. J Epidemiol Community Health 2012; 66:914-8.

36. Figueiredo MCD, Bueno MP, Ribeiro CC, Lima PA, Silva IT. Banco de leite humano: o apoio à amamentação e à duração do aleitamento materno exclusivo. J Hum Growth Dev 2015; 25:204-10. 
37. Programa Nacional das Nações Unidas para o Desenvolvimento. Atlas Brasil. 2016. http:// www.br.undp.org/content/brazil/pt/home/ sustainable-development-goals.html (acessado em 20/Dez/2018).

38. Morrow AL, Guerrero ML, Shults J, Calva JJ, Lutter C, Bravo J, et al. Efficacy of home-based peer counselling to promote exclusive breastfeeding: a randomised controlled trial. Lancet 1999; 353:1226-31.

39. Barros FC, Semer TC, Tonioli Filho S, Victora CG. Avaliação do impacto de centros de lactação sobre padrões de amamentação, morbidade e situação nutricional: um estudo de coorte. Rev Bras Epidemiol 2002; 5:5-14.

40. Sudfeld CR, Fawzi WW, Lahariya C. Peer support and exclusive breastfeeding duration in low and middle-income countries: a systematic review and meta-analysis. PLoS One 2012; 7:e45143.
41. Almeida H, Venancio SI, Sanches MTC, Onuki D. The impact of kangaroo care on exclusive breastfeeding in low birth weight newborns. J Pediatr (Rio J.) 2010; 86:250-3.

42. Departamento de Ações Programáticas Estratégicas, Secretaria de Atenção à Saúde, Ministério da Saúde. Manual do método canguru: seguimento compartilhado entre a atenção hospitalar e a atenção básica. Brasília: Ministério da Saúde; 2015.

43. Sanches MTC, Buccini GS, Gimeno SGA, Rosa TEC, Bonamigo AW. Fatores associados à interrupção do aleitamento materno exclusivo de lactentes nascidos com baixo peso assistidos na atenção básica. Cad Saúde Pública 2011; 27:953-65 


\section{Abstract}

The study aimed to assess factors associated with exclusive breastfeeding in low birthweight infants under six months of age in 64 Brazilian municipalities. This cross-sectional study included the 2,745 low birth weight infants under six months of age in the Survey on Prevalence of Breastfeeding (2008) in Brazilian municipalities. The outcome was exclusive breastfeeding. Individual determinants included socioeconomic characteristics (infants' age and sex), maternal characteristics (age bracket, work status, and parity), and characteristics of health services (birth in a ChildFriendly Hospital; place of outpatient follow-up; type of delivery). Two contextual determinants were also included: "number of Human Milk Banks per municipality per 10,000 live births" and "municipal human development index". The individualized effect of the study factor on the outcome was assessed by multilevel Poisson regression with level of significance under 5\%. Exclusive Breastfeeding was more prevalent in low birth weight infants whose mothers were 20-35 years of age, who were not working away from home or were on maternity leave, who had given birth in a ChildFriendly Hospital, and who lived in municipalities with more human milk banks per 10,000 live births. The result of the positive and independent effect of strategies that were already part of the National Breastfeeding Policy (birth in a ChildFriendly Hospital, living in a municipality with more human milk banks, and adopting laws that guarantee maternity leave) on exclusive breastfeeding in low birth weight infants points to the need to expand the coverage of these measures in order to meet the breastfeeding targets proposed by the World Health Organization.

\section{Low Birth Weight Infant; Breast Feeding; Milk} Banks

\section{Resumen}

El objetivo fue evaluar los factores asociados a la lactancia materna exclusiva en lactantes con bajo peso al nacer menores de seis meses, residentes en 64 municipios brasileños. Este estudio transversal incluyó a los 2.745 lactantes con bajo peso al nacer, menores de seis meses, de la Encuesta de Prevalencia de Lactancia Materna (2008) en municipios Brasileños. El desenlace fue "lactancia materna exclusiva". Los determinantes individuales correspondieron a las características socioeconómicas de los lactantes (edad; sexo), de las madres (franja etaria; situación laboral; igualdad) y de los servicios de salud (nacimiento en un Hospital Amigo da Criança; lugar de seguimiento ambulatorio; tipo de parto). Se incluyeron dos determinantes contextuales: "número de bancos de leche humana por municipio por 10 mil nacidos vivos”, e "IDH del municipio". El efecto individualizado del factor de estudio sobre el desenlace fue evaluado mediante regresión de Poisson con estructura multinivel, considerándose una asociación significante cuando $p<5 \%$. El lactancia materna exclusiva fue más prevalente entre lactantes con bajo peso al nacer, cuyas madres tenian de 20-35 años, no trabajaban fuera o estaban con licencia de maternidad; en quienes nacieron en un Hospital Amigo da Criança y que residian en municipios con mayor número de bancos de leche humana por 10 mil nacidos vivos. El resultado del efecto positivo $e$ independiente de estrategias, que ya integran la Politica Nacional de Lactancia Materna (nacer en Hospital Amigo da Criança, residir en municipio con mayor disponibilidad de bancos de leche humana y adoptar leyes que garanticen la baja por maternidad), sobre el lactancia materna exclusiva en lactantes con bajo peso al nacer, apunta la necesidad de expansión de la cobertura de esas acciones con vistas al cumplimiento de las metas de lactancia propuestas por la Organización Mundial de la Salud.

Recién Nacido de Bajo Peso; Lactancia Materna; Bancos de Leche Humana
Recebido em 07/Jul/2018

Versão final reapresentada em 16/Mar/2019

Aprovado em 22/Mar/2019 